\title{
Gut markers of bodily self-consciousness
}

\author{
Alessandro Monti ${ }^{1,2 *}$, Giuseppina Porciello ${ }^{2,3}$, Maria Serena Panasiti ${ }^{2,3}$, Salvatore M. Aglioti ${ }^{1,2 *}$ \\ ${ }^{1}$ Sapienza, Università di Roma and CLNS@Sapienza, Istituto Italiano di Tecnologia, Rome, \\ Italy.
}

${ }^{2}$ IRCCS Fondazione Santa Lucia research hospital, Rome, Italy.

${ }^{3}$ Dipartimento di Psicologia, Sapienza, Università di Roma, Rome, Italy.

*Correspondence to: alessandro.monti@uniroma1.it or salvatoremaria.aglioti@uniroma1.it.

\section{One sentence summary}

A pill measuring stomach and bowel activity reveals that being aware of one's own body is also a gut feeling.

\footnotetext{
Abstract

Bodily self-consciousness, the state of mind that allows humans to be aware of their own body, forms the backdrop for almost every human experience, yet its underpinnings remain elusive. Here we use an ingestible, minimally invasive capsule and surface electrogastrography to probe 15 if gut physiology correlates with bodily self-consciousness during a virtual bodily illusion. We discover that specific patterns of stomach and large intestine activity (temperature, pressure, $\mathrm{pH}$, and gastric peak frequency) covary with specific facets of bodily self-consciousness (feelings of body ownership, agency, location, and disembodiment). Furthermore, we show that the link between gastro-intestinal parameters and bodily self-consciousness is often moderated by 20 individual levels of interoception. These results reveal a deep visceral pathway to the selfconscious perception of ourselves as embodied beings.
} 
Humans are ordinarily aware that their body is part and parcel of their self $(1,2)$. Physiological, clinical, and behavioral data suggest that bodily self-consciousness arises when the central nervous system integrates pieces of body-related information coming from the external and internal senses in a coherent fashion $(3,4)$. While the contribution of sight, touch, heartbeats and breathing to corporeal awareness is now ascertained (5-8), the role of deep, sub-diaphragmatic organs is unknown. Despite their potential significance for higher order cognitive functions (9), organs located in the abdominal cavity, such as the stomach and the intestine, are difficult to reach and probe without relying on invasive methods $(10,11)$. somatosensory and insular cortices. In turn, this activity is modulated by top-down control exerted by central and enteric neurons (14). While the gut-brain loop has the clear homeostatic purpose of regulating food intake, it has been proposed that gut signals, once in the brain, may also influence a variety of higher-order processes, including corporeal awareness $(15,16)$. Gut physiology per se is rarely attended to or perceived at the conscious level. Nevertheless, we reasoned that ongoing gut activity could provide the brain with the kind of internal information needed to give us the normal, everyday sensation that our body is not empty.

To shed light on the visceral roots of bodily self-consciousness, we asked healthy participants ( $N$ $=31$ ) to undergo a simplified version of the new embreathment illusion $(8)$ after they ingested a wireless capsule (SmartPill ${ }^{\mathrm{TM}}$ ) fitted with sensors tracking temperature, pressure, and $\mathrm{pH}$ values across the entire gastro-intestinal tract in real time (17). We simultaneously monitored the gastric contractions of our subjects through surface electrogastrography (EGG) (18). Combining these methods, we measured gut physiological parameters in two experimental conditions, in which 
participants embodied a virtual body (avatar) that looked, lay, and breathed like them (congruent condition) or not (incongruent condition) (Fig. 1). To assess if and how each district of the gastro-intestinal tract relates to bodily self-consciousness, we administered the illusion thrice: first when the SmartPill was in the stomach, then after the capsule entered the small bowel, and finally when it reached the large bowel. We hypothesized that objective gut signals recorded during the experimental conditions would predict the subjective feelings of owning, controlling, or dwelling into a body, as gauged through a visual analogue scale (VAS) questionnaire (8). We also expected that these effects varied according to objective (19) and subjective (20) levels of interoception, i.e. the perception of inner physiological signals (21) (see Supplementary Materials below the "Download PDF" link in the bioRxiv webpage for details).

We found that the higher the temperature of the stomach and the $\mathrm{pH}$ of the large intestine, the more our participants reported to feel a sense of ownership towards a congruent virtual body that looked, lay, and breathed like them, specifically if they had a below-average level of interoceptive accuracy (Fig. 2; see also Fig. S5). For each $1^{\circ} \mathrm{C}$ increase in stomach temperature, there was a predicted $62.62 \pm 29.8$ points increase in VAS ratings of body ownership $(t=2.104$ $p=0.0471)$. For each 1-unit increase in large intestine $\mathrm{pH}$, there was a predicted $19.51 \pm 9.03$ points increase in the same scale of perceived body ownership $(t=2.160, p=0.0414)$.

Likewise, the higher the temperature and the pressure of the stomach, the less our participants were inclined to feel disembodied, i.e. to feel like they had no body - an effect moderated also by their interoceptive abilities and by the congruency between the virtual and the real body (Fig.

3A and 3C). At average levels of interoceptive accuracy, for each $1{ }^{\circ} \mathrm{C}$ increase in stomach temperature, predicted drops in VAS disembodiment ratings were $-41.78 \pm 15.7$ for human-like virtual bodies $(t=-2.625, p=0.0164)$ and $-36.09 \pm 13.7$ for non-human-like virtual bodies $(t=-$ 
$2.625, p=0.0164)$. For each $1 \mathrm{mmHg}$ increase in stomach pressure, predicted VAS points drops were $-40.291 \pm 19.14$ (interoceptive sensibility: below average, $t=2.105, p=0.0435$ ), $-24.142 \pm$ 8.61 (interoceptive sensibility: average, $t=-2.803, p=0.0087$ ), $-22.435 \pm 7.79$ (interoceptive accuracy: average, $t=2.878, p=0.0072$ ), $-38.573 \pm 16.44$ (interoceptive accuracy: above average, $\mathrm{t}=-2.346, p=0.0256$ ). Furthermore, when people with good interoception observed an incongruent virtual body, the higher the stomach $\mathrm{pH}$, the less they felt disembodied $(-55.50 \pm$ 14.22 VAS points, $t=-3.904, p<0.001$ ), while the effect was reversed in people with poor interoception (52.46 \pm 23.44 VAS points, $t=2.238, p=0.0327$; Fig. 3B).

Gastric activity correlated not only with perceived body ownership and disembodiment, but also with the two other facets of bodily self-consciousness, namely, the sense of agency and the sense of location. The higher the stomach pressure of participants with average and below-average interoception, the stronger their sense of agency over an incongruent avatar (interoceptive sensibility: average, $22.180 \pm 9.81$ points, $t=2.262, p=0.0291$; below-average, $45.536 \pm 21.81$ points, $t=2.088, p=0.0431$; Fig. 4A). Moreover, the higher the stomach $\mathrm{pH}$, the more our participants felt localized into an incongruent avatar (interoceptive accuracy: average, $22.71 \pm$ 10.31 points, $t=2.204, p=0.0372$; Fig. 4B).

Capitalizing on the smart pills technology to wirelessly monitor the physiological parameters of the gastro-intestinal tract in real time while healthy subjects underwent a bodily illusion in immersive virtual reality, we were able to predict individual ratings of bodily self-consciousness from individual patterns of gut activity. According to our results, each class of gut signals, particularly of those recorded in the stomach, exerts a quite specific role within the framework of bodily self-consciousness: stomach temperature is a marker of perceived ownership and stomach pressure has a special relationship with agency, while $\mathrm{pH}$ is tied to the senses of location and 
ownership. In parallel, when any of these three signals goes up, participants feel less disembodied, particularly when their virtual body does not match their real body. Hence, to an extent, gastro-intestinal cues can override conflicting visual, spatial, and respiratory information and therefore reduce the sense of disembodiment. We speculate that this compensatory process is impaired in psychiatric conditions that alter bodily self-consciousness, as in eating disorders and depersonalization.

In contrast, an uptick in the number of stomach contractions, as measured through EGG peak frequency, affects bodily self-consciousness as a whole, in a less specific fashion (Fig. S5). Thus, compared to EGG, smart pills offer a deeper, clearer picture of the links between gut physiology and the bodily self, or between gut physiology and mental states in general. This feature could make the smart pills and the increasingly sophisticated ingestibles that will likely appear in the future $(22,23)$ a particularly valuable tool for assessing also other important psychological constructs that may be rooted in gastro-intestinal physiology, from emotions (24) to moral disgust $(25,26)$, in a minimally invasive way. periphery is linked to one's sense of oneself as an embodied entity. More studies actively stimulating the gastro-intestinal tract are needed to clarify whether this link has a causal nature. If so, this would imply that bodily self-consciousness is based upon multisensory integration processes whose scope is even wider than previously thought - processes gathering a wealth of sensory information from abdominal viscera as well as from thoracic organs and from skin receptors. Such a diverse range of sensory inputs probably helps the brain to maintain a 'body template' in a variety of circumstances, including those in which a particular sensory channel may be temporarily or permanently shut down, as in darkness, blindness, or spinal cord injuries. 


\section{References and notes}

1. G. Berlucchi, S. M. Aglioti, The body in the brain revisited. Experimental Brain Research. 200, 25-35 (2010).

2. W. James, The principles of psychology (New York : Holt, 1890).

3. O. Blanke, M. Slater, A. Serino, Behavioral, Neural, and Computational Principles of Bodily Self-Consciousness. Neuron. 88, 145-166 (2015).

4. H.-D. Park, O. Blanke, Coupling Inner and Outer Body for Self-Consciousness. Trends in Cognitive Sciences. 23, 377-388 (2019).

5. H. H. Ehrsson, The experimental induction of out-of-body experiences. Science. 317, 1048 (2007).

6. B. Lenggenhager, T. Tadi, T. Metzinger, O. Blanke, Video Ergo Sum: Manipulating Bodily Self-Consciousness. Science. 317, 1096-1099 (2007).

7. H.-D. Park, F. Bernasconi, J. Bello-Ruiz, C. Pfeiffer, R. Salomon, O. Blanke, Transient Modulations of Neural Responses to Heartbeats Covary with Bodily Self-Consciousness. $J$. Neurosci. 36, 8453-8460 (2016).

8. A. Monti, G. Porciello, G. Tieri, S. M. Aglioti, The "embreathment" illusion highlights the role of breathing in corporeal awareness. Journal of Neurophysiology. 123, 420-427 (2019).

9. M. Schemann, T. Frieling, P. Enck, To learn, to remember, to forget-How smart is the gut? Acta Physiologica. 228, e13296 (2020). 
10. Y. Y. Lee, A. Erdogan, S. S. C. Rao, How to assess regional and whole gut transit time with wireless motility capsule. Journal of Neurogastroenterology and Motility. 20, 265-270 (2014).

11. R. J. Saad, The Wireless Motility Capsule: a One-Stop Shop for the Evaluation of GI Motility Disorders. Current Gastroenterology Reports. 18 (2016).

12. B. D. Umans, S. D. Liberles, Neural Sensing of Organ Volume. Trends in Neurosciences. 41, 911-924 (2018).

13. M. M. Kaelberer, K. L. Buchanan, M. E. Klein, B. B. Barth, M. M. Montoya, X. Shen, D. V. Bohórquez, A gut-brain neural circuit for nutrient sensory transduction. Science. 361 (2018), doi:10.1126/science.aat5236.

14. J. B. Furness, B. P. Callaghan, L. R. Rivera, H.-J. Cho, in Microbial Endocrinology: The Microbiota-Gut-Brain Axis in Health and Disease, M. Lyte, J. F. Cryan, Eds. (Springer, New York, NY, 2014), pp. 39-71.

15. I. Rebollo, A.-D. Devauchelle, B. Béranger, C. Tallon-Baudry, Stomach-brain synchrony reveals a novel, delayed-connectivity resting-state network in humans. eLife. 7, e33321 (2018).

16. G. Porciello, A. Monti, S. M. Aglioti, How the stomach and the brain work together at rest. eLife. 7, e37009 (2018).

17. R. J. Saad, W. L. Hasler, A technical review and clinical assessment of the wireless motility capsule. Gastroenterol Hepatol (N Y). 7, 795-804 (2011). 
18. J. Yin, J. D. Z. Chen, Electrogastrography: Methodology, Validation and Applications. J Neurogastroenterol Motil. 19, 5-17 (2013).

19. R. Schandry, Heart beat perception and emotional experience. Psychophysiology. 18, 483488 (1981).

20. G. Calì, E. Ambrosini, L. Picconi, W. E. Mehling, G. Committeri, Investigating the relationship between interoceptive accuracy, interoceptive awareness, and emotional susceptibility. Front Psychol. 6, 1202 (2015).

21. A. Monti, G. Porciello, M S. Panasiti, S. M. Aglioti, The inside of me: interoceptive constraints on the concept of self in neuroscience and clinical psychology. Psychological Research. In press.

22. M. Mimee, P. Nadeau, A. Hayward, S. Carim, S. Flanagan, L. Jerger, J. Collins, S. McDonnell, R. Swartwout, R. J. Citorik, V. Bulović, R. Langer, G. Traverso, A. P. Chandrakasan, T. K. Lu, An ingestible bacterial-electronic system to monitor gastrointestinal health. Science. 360, 915-918 (2018).

24. E. P. M. Vianna, D. Tranel, Gastric myoelectrical activity as an index of emotional arousal. International Journal of Psychophysiology. 61, 70-76 (2006).

25. P. Rozin, J. Haidt, K. Fincher, From Oral to Moral. Science. 323, 1179-1180 (2009).

26. K. J. Eskine, N. A. Kacinik, J. J. Prinz, A bad taste in the mouth: gustatory disgust influences moral judgment. Psychol Sci. 22, 295-299 (2011). 
27. O. A. Al-Shboul, The Importance of Interstitial Cells of Cajal in the Gastrointestinal Tract. Saudi J. Gastroenterol. 19, 3-15 (2013).

28. J. D. Huizinga, W. J. E. P. Lammers, Gut peristalsis is governed by a multitude of cooperating mechanisms. Am. J. Physiol.-Gastrointest. Liver Physiol. 296, G1-G8 (2009). heart: distinguishing interoceptive accuracy from interoceptive awareness. Biol. Psychol. 104, 65-74 (2015).

30. K. L. Koch, R. M. Stern, Eds., Handbook of Electrogastrography (Oxford University Press, Oxford, New York, 2003). Advanced Analysis of MEG, EEG, and Invasive Electrophysiological Data. Comput. Intell. Neurosci. 2011, 1-9 (2011).

32. D. M. Bates, M. Mächler, B. Bolker, S. Walker, Fitting Linear Mixed-Effects Models Using lme4. J. Stat. Softw. 67, 1-48 (2015). Mixed Effects Models. J. Stat. Softw. 82 (2017).

34. P. C. D. Johnson, Extension of Nakagawa \& Schielzeth's R2GLMM to random slopes models. Methods Ecol. Evol. 5, 944-946 (2014).

35. A. Gelman, J. Hill, Data Analysis Using Regression and Multilevel/Hierarchical Models (Cambridge University Press, 2007). 


\section{Acknowledgements}

We thank Danila Cosenza for medical assistance and Maurizio Molisso for standardized meal preparation. Figures 1, S2, and S3 were designed with BioRender.com. Funding: Supported by ERC Advanced Grant no. 789058. Author contributions: Conceptualization and methodology:

M.S.P. Funding acquisition, project administration, resources, supervision: S.M.A. Software:

A.M. Writing - original draft: A.M. Writing - review \& editing: A.M., G.P., M.S.P. and S.M.A. Competing interests: authors declare no competing interests. Data and materials availability: see data and materials at https://osf.io/wecta/?view_only=45e1a9e30c2a47efa82c10161b70b732. 

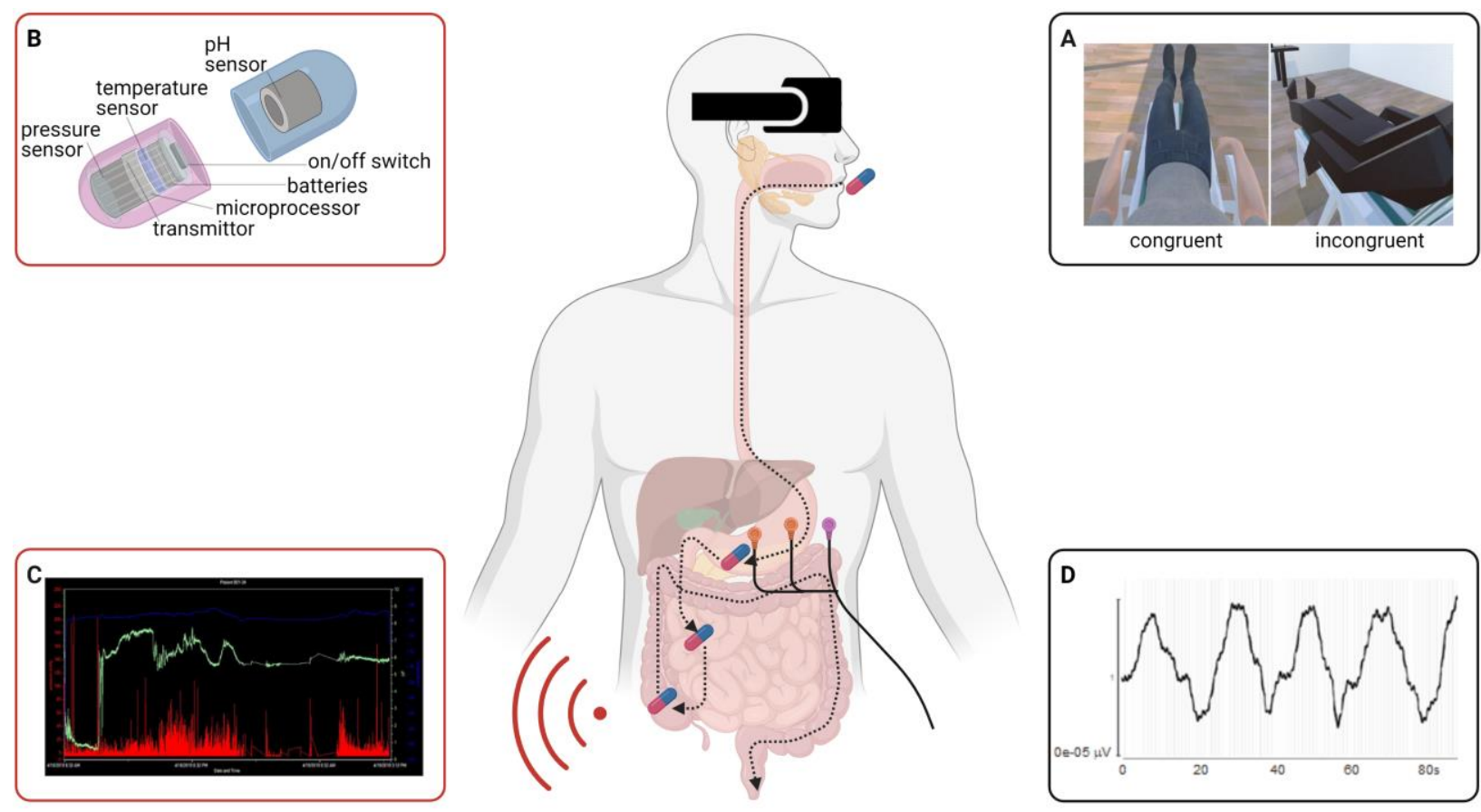

Figure 1. Experimental methods. Participants are administered a simplified 'embreathment' bodily illusion (A) in which they embody an avatar that looks, lays, and breathes like them (congruent condition) or not (incongruent condition). While they undergo the illusion, their gut is monitored through the SmartPill (capsule icon), which is fitted with a variety of sensors (B). This is done three times, as the pill moves down the three main gut segments (stomach, small bowel, and large bowel). The first time, since the capsule lies in the stomach, Smart Pill measures are complemented by electrogastrography (electrode icons). A standard SmartPill graph (C) plots temperature (blue line), $\mathrm{pH}$ (green line), and pressure (red bars) across the different segments of the gastro-intestinal tract over time. A typical electrogastrographic recording (D) shows gastric myoelectric activity generated by interstitial cells of Cajal. Inset (B) is adapted from (10). 


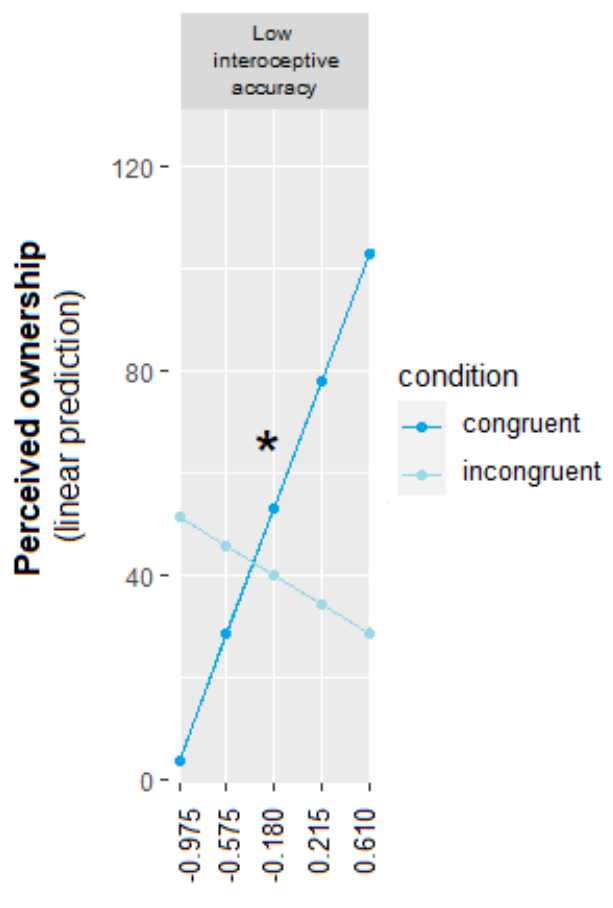

Stomach $\mathrm{T}\left({ }^{\circ} \mathrm{C}\right)$

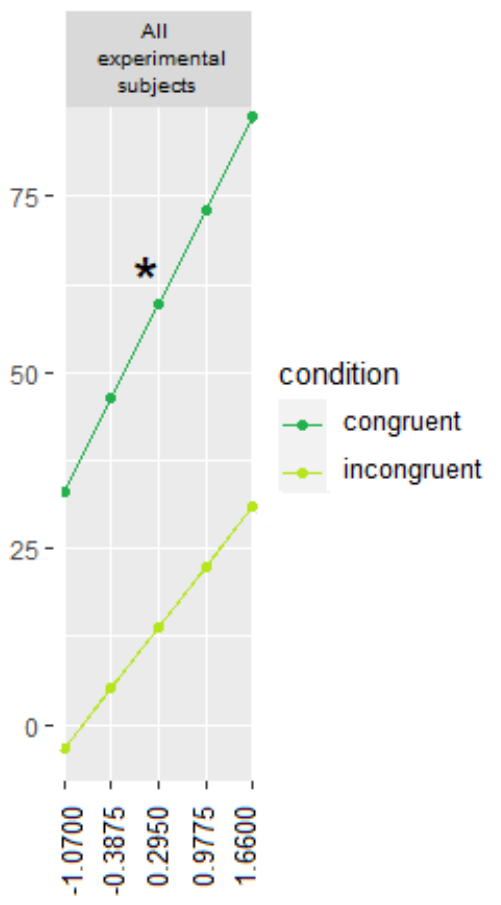

Large bowel pH

Figure 2. Effects of stomach temperature (left) and large intestine $\mathrm{pH}$ (right) on perceived body ownership (estimated marginal means). 'Low interoceptive accuracy' indicates that the effects shown in the left graph occur when interoceptive accuracy (19) is 1 standard deviation below the mean. 'All experimental subjects' indicates that the specific relation between large intestine $\mathrm{pH}$ and sense of body ownership holds across the entire pool of participants. However, the overall impact of large intestine $\mathrm{pH}$ on bodily self-consciousness is stronger when interoception is low (see Figure S5). 


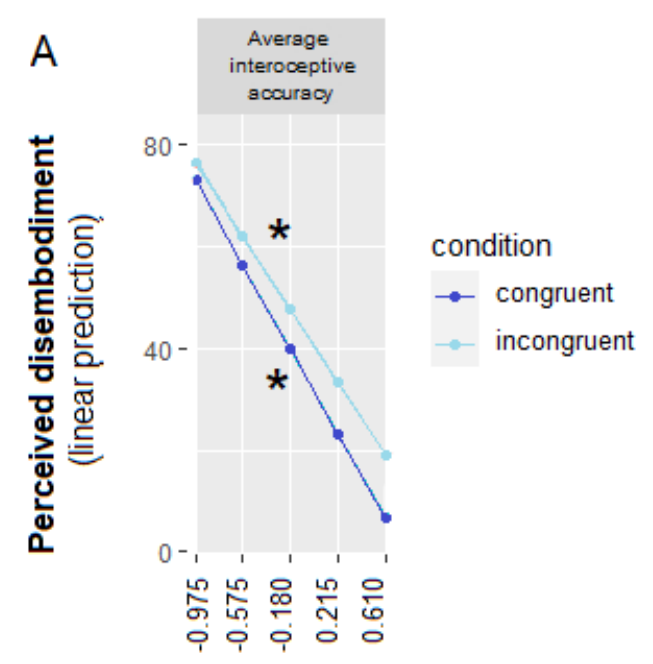

Stomach $\mathrm{T}\left({ }^{\circ} \mathrm{C}\right)$
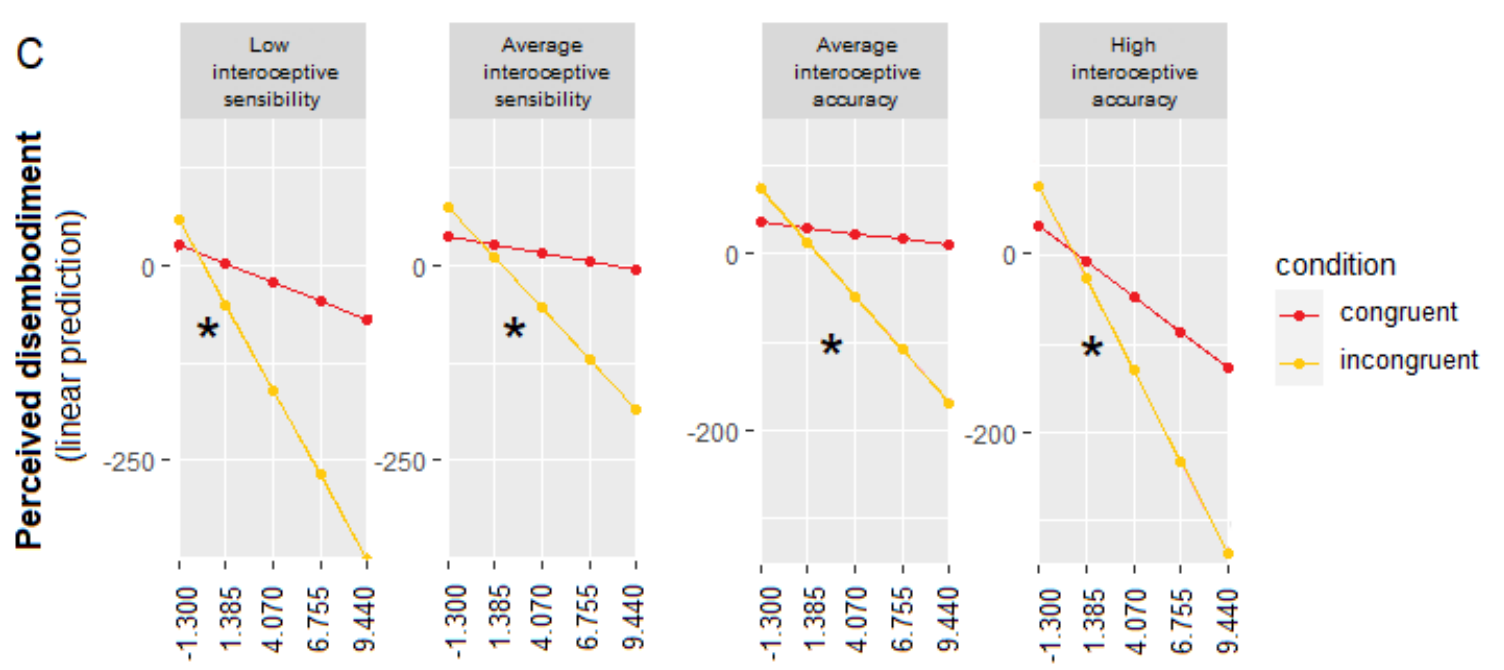

Stomach pressure $(\mathrm{mmHg})$

Figure 3. Effects of stomach temperature (A), $\mathrm{pH}(\mathrm{B})$, and pressure (C) on perceived disembodiment (estimated marginal means). 'Low' accuracy as in Figure 2. 'Average' and 'high' accuracy mean that the effects shown below these labels occur when interoceptive accuracy is equal to the mean or 1 S.D. above the mean, respectively. 'Sensibility' is self-reported interoception (see Supplementary Materials). 


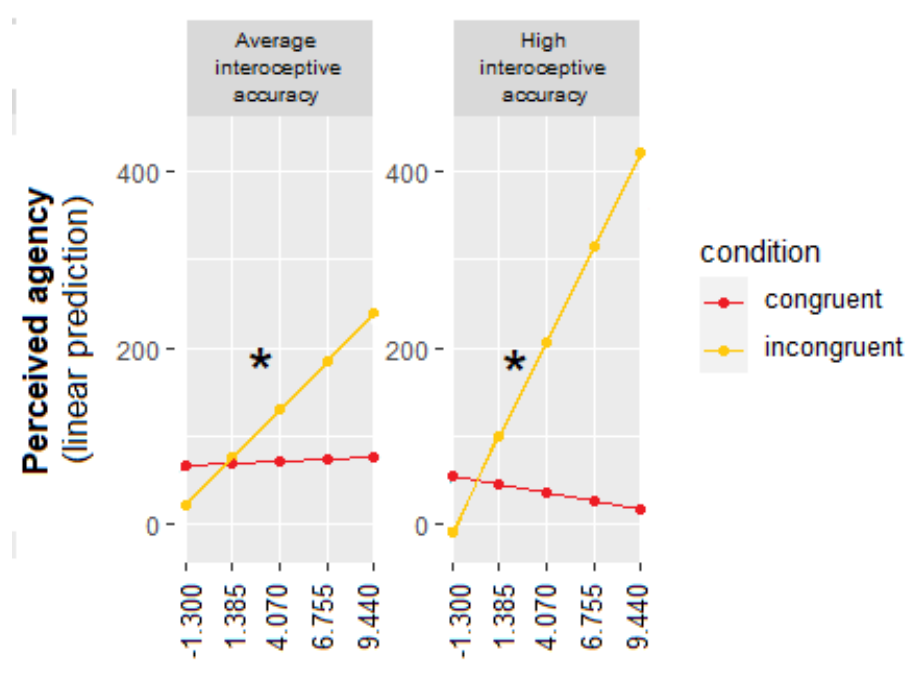

Stomach pressure $(\mathrm{mmHg})$

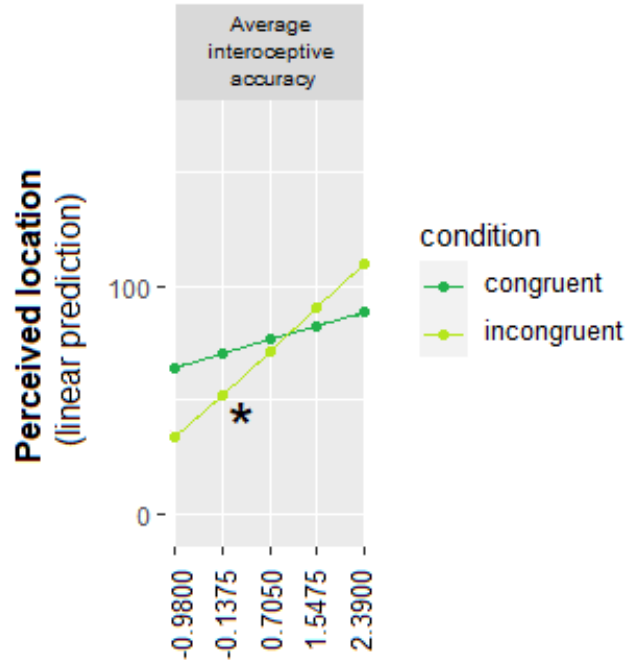

Stomach pH

Figure 4. Effects of stomach pressure (A) and $\mathrm{pH}(\mathrm{B})$ on ratings of body agency and location (estimated marginal means). 'Low', 'average' and 'high' accuracy as in Figures 2 and 3. 